\title{
Erratum to: Monte Carlo simulations of a disordered superconductor-metal quantum phase transition ${ }^{\star}$
}

Eur. Phys. J. B (2018) 91: 311, https://doi.org/10.1140/epjb/e2018-90497-5

Ahmed K. Ibrahim and Thomas Vojta ${ }^{\mathrm{a}}$

Department of Physics, Missouri University of Science and Technology, Rolla, MO 65409, USA

Received 21 March 2019

Published online 10 April 2019

(C) EDP Sciences / Società Italiana di Fisica / Springer-Verlag GmbH Germany, part of Springer Nature, 2019

Figure 9 in the published article was not correct. This erratum provides correct Figure 9:

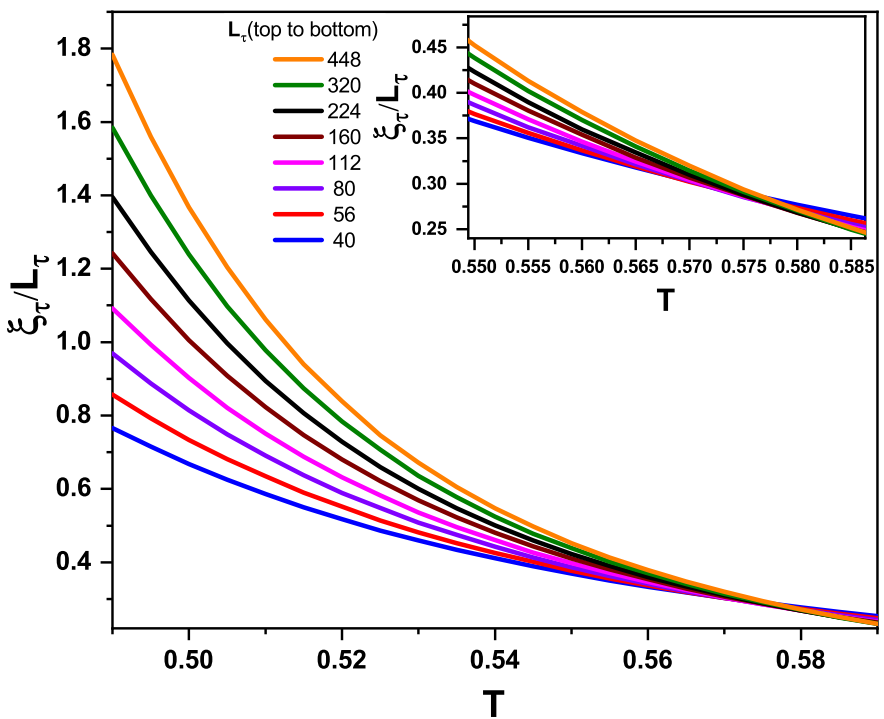

Fig. 9. Scaled correlation time $\xi_{\tau} / L_{\tau}$ versus temperature $T$ for different values of $L_{\tau}$ in the Griffiths region. The system size in space is $L=1000$; the data are averaged over 2000 disorder configurations. The inset shows a magnification for the crossing point of the curves.

The Publisher apologizes for the inconvenience.

\footnotetext{
${ }^{\star}$ The online version of the original article can be found at https://doi.org/10.1140/epjb/e2018-90497-5

a e-mail: vojtat@mst.edu
} 